EDITORIAL

\title{
Should ablation be the first line treatment for supraventricular arrhythmias?
}

\section{F G Cosío}

Heart 2005;91:5-6. doi: 10.1136/hrt.2004.040121

Catheter ablation remains a good first line choice for the palliative treatment of symptomatic typical atrial flutter

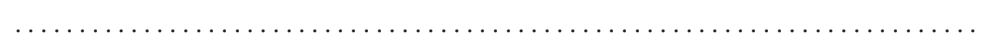

$\mathrm{R}$ adiofrequency (RF) catheter ablation was first used in the 1990s and completely changed the management of supraventricular tachycardias. After about 20 years of development, clinical electrophysiological methods provided accurate knowledge of tachycardia mechanisms that led first to successful surgical treatment, then to exquisite anatomical localisation, allowing endocardial catheter ablation. Clear separation of the mechanisms into focal discharge, accessory pathway dependent or intranodal re-entry was an essential step and, during this process, the general term "supraventricular", encompassing multiple mechanisms, started to lose some of its meaning.

Perhaps the paradigm of catheter ablation effectiveness is Wolff-Parkinson-White syndrome, where a congenital structural abnormality, ${ }^{1}$ the accessory atrioventricular (AV) pathway, is fully responsible for the disease mechanisms. Once the anomaly is identified, intracardiac mapping can locate the anomaly with great precision; then RF ablation can induce a localised necrosis that affects the accessory pathway, eliminating its functional consequences $^{2-4}$ with very low morbidity and mortality. ${ }^{5-7}$ Not only does RF ablation produce a complete cure, but it also provides experimental proof of the pathogenic working diagnosis, as it completely prevents recurrence of AV re-entrant tachycardias. Nevertheless, atrial fibrillation (AF) can recur in some patients, particularly in older individuals, ${ }^{8}$ when the accessory pathway is not the only cause of AF.

\section{NODAL RE-ENTRANT TACHYCARDIA}

This clear cut view cannot be wholly applied to all supraventricular tachycardias. In nodal reentrant tachycardia, despite a very high rate of success and an important research effort, it is not completely clear if it is ablation of the AV node extensions, ${ }^{10}$ or the intermediate AV nodal tissue, or even the myocardium around the tricuspid ring showing nodal-like electrophysiological properties that interrupts the circuit. ${ }^{11}$ In focal atrial tachycardia a lower efficacy rate and a higher recurrence rate $^{7}$ probably reflects our incomplete knowledge of the mechanism and the anatomic bases. Recent work by the Taipei group has disclosed some unique features, such as channelling of conduction for some distance from the focus to the point where activation
Spain; fcosio@vitanet.nu
Getafe, Carretera de spreads throughout the atria, and the anatomic and functional bases for this peculiar path of activation are unknown. ${ }^{12}$ Even so, in atrial tachycardia ablation can eliminate the focus of origin of the abnormal activation and prevent tachycardia recurrence.

\section{ABLATION OF ATRIAL FLUTTER}

Ablation of typical atrial flutter is quite a different proposition in several aspects. We are very successful at interrupting the arrhythmia and preventing its recurrence, but we do not know what its cause is. Practically all epidemiological data have lumped flutter and fibrillation together and we are ignorant of what may make some $10 \%$ of these mixed populations have atrial flutter and the others AF, nor do we have any anatomic or functional data that let us separate both populations or clarify why flutter occurs more in men than women by a ratio of 3:1. The inferior vena cava-tricuspid isthmus has become the universally accepted target for ablation of the typical flutter circuit, due to its accessibility and because it is located far from the AV node, even though we have no evidence that this site is the cause of atrial flutter. The relatively slower conduction described through this isthmus ${ }^{13}$ in relation to the anterior right atrial wall is also found in a control population without flutter and probably depends on fibre architecture and anisotropic conduction. ${ }^{14}{ }^{15}$ Ablation and interruption of isthmus conduction does nothing to the disease mechanisms that cause flutter in the first place and, if indeed the causes of flutter and fibrillation are the same, basic progression to later development of atrial fibrillation should be expected. ${ }^{16}$

However, clinically atrial flutter can be difficult to manage. If it is the initial presenting sign or the result of drug treatment for $\mathrm{AF}$, flutter is a troublesome arrhythmia, often poorly tolerated because of a rapid, difficult to control, AV conduction rate. The efficacy of isthmus ablation is well established and its safety record outstanding. ${ }^{17-20}$ The procedure is therefore applicable in almost any clinical circumstance, which by itself would make catheter ablation a good choice as first line treatment. If AF occurs later, it can be controlled more easily in most cases.

The report in this issue of Heart by Da Costa and colleagues ${ }^{21}$ agrees with previous experience in regard to a low rate of flutter recurrence and other atrial tachycardia recurrences-it is the new atrial fibrillation occurrence rate around

Abbreviations: $A F$, atrial fibrillation; $A V$, atrioventricular; $R F$, radiofrequency 
$15 \%$ that could not be affected by flutter ablation. Flutter could be an inducer of fibrillation through shortening and dispersion of refractory periods by the fast atrial rate, an effect described by Wijffels and others as electrical remodelling, ${ }^{22} 23$ and a potential cause for perpetuation of atrial fibrillation. But even though this may play a role in some patients in whom flutter ablation prevents further episodes of fibrillation, ${ }^{24}$ the data presented by Da Costa and colleagues ${ }^{21}$ suggest that this may not be so important, as delaying ablation by an average of two years did not have a detrimental effect. Nevertheless, the patients undergoing earlier ablation probably were more likely to not need antiarrhythmic drugs or anticoagulants, and suffer less tachycardia episodes during that time, which could have been a worthwhile benefit by itself.

\section{LACK OF PREVENTIVE STRATEGY}

The problem with our present management of atrial flutter and $\mathrm{AF}$ is the lack of a clear, long term primary and secondary preventive strategy. Both isolated drug treatment or catheter ablation of atrial flutter may be the equivalent of administering nitrates and $\beta$ blockers to a patient with effort angina, without paying any attention to his hypertension, high cholesterol, or smoking 40 cigarettes per day. In contrast with accessory pathways, and probably also with nodal reentrant tachycardia, even though flutter ablation terminates the arrhythmia and prevents its recurrence, it does not stop progression of whatever basic atrial disease caused it. Prevention of AF will depend more on identifying these pathogenic mechanisms and learning to contain or revert atrial myocardial damage. In the meantime, given its efficacy and safety, catheter ablation remains a good first line choice for the palliative treatment of symptomatic typical atrial flutter.

\section{REFERENCES}

1 Becker AE, Anderson RH, Durrer D, et al. Anatomical substrates of WolffParkinson-White syndrome. A clinicopathologic correlation in seven patients. Circulation 1978:57:870-9.

2 Kuck KH, Schlüter M, Ageiger $M$, et al. Radiofrequency catheter ablation of accessory atrioventricular pathways. Lancet 1991;337:1557-61.

3 Jackman WM, Wang X, Friday KJ, et al. Catheter ablation of accessory atrioventricular pathways (Wolff-Parkinson-White syndrome) by radiofrequency current. N Engl J Med 1991;324:1605-12.

4 Calkins H, Sousa J, El-Atassi R, et al. Diagnosis and cure of the WolffParkinson-White syndrome or paroxysmal supraventricular tachycardia during a single electrophysiologic test. N Engl J Med 1991;324:1612-8.
5 Hindricks G. The multicentre European radiofrequency survey (MERFS): complications of radiofrequency catheter ablation of arrhythmias. Eur Heart $J$ 1993; 14:1644-53

6 Chen S-A, Chiang C-E, Tai C-T, et al. Complications of diagnostic electrophysiologic studies and radiofrequency catheter ablation in patients with tachyarrhythmias: an eight-year survey of 3,966 consecutive procedures in a tertiary referral center. Am J Cardiol 1996;77:41-6.

7 Scheinman MM, Huang S. The 1998 NASPE prospective catheter ablation registry. PACE 2000;23:1020-8.

8 Weiss R, Knight BP, Bahu M, et al. Long-term follow-up after radiofrequency ablation of paroxysmal supraventricular tachycardia in patients with tachycardia-induced atrial fibrillation. Am J Cardiol 1997;80:1609-10.

9 Dagres N, Clague JR, Kottkamp H, et al. Radiofrequency catheter ablation of accessory pathways; outcome and use of antiarrhythmic drugs during followup. Eur Heart J 1999;20:1826-32.

10 Inoue S, Becker AE. Posterior extensions of the human compact atrioventricular node: a neglected anatomic feature of potential clinical significance. Circulation 1998:97:188-93.

11 McGuire MA, DE Bakker JMT, Vermeulen JT, et al. Atrioventricular junctional tissue: discrepancy between histological and electrophysiological characteristics. Circulation 1996;94:571-7.

12 Higa S, Tai C-T, Lin Y-J, et al. Focal atrial tachycardia. New insight from noncontact mapping and catheter ablation. Circulation 2004;109:84-91.

13 Tai C-T, Chen S-A, Chiang C-E, et al. Characterization of low right atrial isthmus as the slow conduction zone and pharmacological target in typical atrial flutter. Circulation 1997;96:2601-11.

14 Cabrera JA, Sanchez-Quintana D, Ho SY, et al. The architecture of the atrial musculature between the orifice of the inferior caval vein and the tricuspid valve: the anatomy of the isthmus. J Cardiovasc Electrophys 1998;14:1186-95.

15 Waki K, Saito T, Becker AE. Right atrial flutter isthmus revisited: normal anatomy favors nonuniform anisotropic conduction. J Cardiovasc Electrophys 2000;11:90-4.

16 Biblo LA, Yuan Z, Quan KJ, et al. Risk of stroke in patients with atrial flutter. Am J Cardiol 2001;87:346-8.

17 Fischer B, Jaïs $P$, Shah $D$, et al. Radiofrequency catheter ablation of common atrial flutter in 200 patients. J Cardiovasc Electrophysiol 1996;7:1225-33.

18 Tsai C-F, Tai C-T, Yu W-C, et al. Is 8-mm more effective than 4-mm tip electrode catheter for ablation of typical atrial flutter? Circulation 1999;100:768-71

19 Cosío FG, López-Gil M, Arribas F, et al. Ablación de flutter auricular. resultados a largo plazo tras 8 años de experiencia. Revista Española de Cardiología 1998;51:832-9.

20 Anselme F, Saoudi N, Poty H, et al. Radiofrequency catheter ablation of common atrial flutter: significance of palpitations and quality-of-life evaluation in patients with proven isthmus block. Circulation 1998;99:534-40.

21 Da Costa A, Romeyer-Bouchard C, Zarqane-Sliman N et al. Impact of first line radiofrequency ablation in patients with lone atrial flutter on the long term risk of subsequent atrial fibrillation. Heart 2005;91:97-8.

22 Wiiffels MCEF, Kirchhof CJHJ, Dorland R, et al. Atrial fibrillation begets atrial fibrillation. A study in awake chronically instrumented goats. Circulation 1995;92:1954-68.

23 Fareh S, Villemarie C, Nattel S. Importance of refractoriness heterogeneity in the enhanced vulnerability to atrial fibrillation induction caused by tachycardia-induced atrial electrical remodeling. Circulation 1998:98:2202-9.

24 Haïssaguerre $\mathrm{M}$, Marcus Fl, Fischer B, et al. Radiofrequency catheter ablation in unusual mechanisms of atrial fibrillation: report of three cases. J Cardiovasc Electrophysiol 1994;5:743-51 